Communications in Physics, Vol. 25, No. 4 (2015), pp. 317-325

DOI:10.15625/0868-3166/25/4/6887

\title{
THERMOELASTIC DAMPING DEPENDING ON VIBRATION MODES OF NANO BEAM RESONATOR
}

\author{
CHU MANH HOANG \\ International Training Institute for Materials Science, \\ Hanoi University of Science and Technology, \\ No. 1, Dai Co Viet, Hai Ba Trung, Hanoi, Vietnam
}

Received 4 September 2015

Accepted for publication 15 December 2015

E-mail: hoangcm@itims.edu.vn

\begin{abstract}
The obtainable quality factor for a nano beam resonator is limited due to internal damping such as thermoelastic damping. Therefore, understanding how internal damping varies with the respective resonant modes is very important to design a high performance nanoresonator. In this research, we investigate thermoelastic damping depending on vibration modes of nano beam resonators using finite element method. The study results show that the quality factor of a nanoresonator is lower than at high order modes. The silicon nano beam resonator with the quality factor larger than one million can be achieved by optimizing the dimensions of the resonant beam.
\end{abstract}

Keywords: Nano beam resonator, internal damping, operation mode, thermoelastic damping, FEM..

\section{INTRODUCTION}

Micro-and nano-mechanical beam resonators are promising for applications in timing, sensing, and physical measurement [1-5]. The advantages of micro- and nano-resonators over the macro-size ones are high operation frequency, high sensitivity in physical measurements, and low energy consumption. The requirement of a resonator for such applications is high quality factor. A resonator with high $\mathrm{Q}$ supplies high sensitivity, low noise, and low energy consumption. Besides high $\mathrm{Q}$, the resonators also requires to operate at high frequency, for example, to enhance the time resolution of measurement in atomic force microscopy and real-time measurements [6]. In order to increase the resonant frequency, the size of the resonator needs to be decreased. However, this leads to high surface to volume ratio, the air damping becomes high in the micro-and nano-beam resonators. In order to maximize the quality factor, it is fundamental to understand the physical mechanisms limiting the quality factor. Physical mechanisms of energy dissipation can be described by the two following sets of losses: extrinsic and intrinsic. The extrinsic losses include acoustic radiation, surface loss, and air friction. The intrinsic losses include material loss, anchor loss, and thermoelastic damping. Under low vacuum condition, losses in such resonators are often generated from extrinsic damping mechanisms such as air damping [7]. Under high vacuum condition, extrinsic damping mechanisms becomes negligible, intrinsic damping mechanisms 
such as attachment loss and thermoelastic damping (TED) need to be taken into account. Thermoelastic damping causes internal energy dissipation that is considered a significant energy loss mechanism affecting the upper limitation of the quality factor of a nanoresonator at room temperature [8]. Thermoelastic damping models have been introduced for investigating the quality factor of different resonant structures such as micro-gyroscopes, bulk resonators, plate type and beam resonators [8-12]. The resonators with high quality factor can be obtained by optimizing designs and/or operating the device at low temperature for reducing thermoelastic damping [13-15]. The thermoelastic damping relates to the irreversible process of heat flow generated by extension and compression regions in the vibration structure. The compression in the beam leads to a higher temperature region while the tensile stress on the opposite side of the beam results in a lower temperature region. In general, the strength of damping caused by TED depends on the vibration frequency, the relevant thermal relaxation time constant, and on the stress distribution in the structure. These, in turn, depend on the structural geometry and also on the material properties which are themselves functions of temperature. The largest loss causing by thermoelastic damping occurs when the period of vibration and the thermal relaxation time are of the same order. Since heat flow is an irreversible process, this heat flow will be associated with energy loss from the vibration mode, and corresponding damping for the resonant mode. Moreover, recently, the operation in ambient air/fluid at high order modes has been employed in order to improve the quality factor of nano-scale resonators [16-18]. Higher-mode oscillation can improve the quality factor due to the relatively lower surface to bulk ratio, which leads to lower air/fluid damping. Consequently, a study on thermoelastic damping depending on the operation modes of the nanoresonators is necessary. However, previous studies mainly concentrate on investigating the thermoelastic damping of flexible fundamental modes [8-13]. Moreover, there are not many reports on nano beam resonators with ultrahigh quality factor in considering influence of thermoelastic damping.

In this paper, we therefore focus on investigating the thermoelastic damping depending on the operation modes of nano beam resonator. It is assumed that the damping mechanism in the resonator is dominant by the thermoelastic damping. The quality factor of the resonator which varies with the geometrical parameters of the resonator is investigated. This study is also motivated to predict the ultimate $\mathrm{Q}$ value that a nano beam resonator can achieve.

\section{MODEL FOR ANALYSIS}

The quality factor $\mathrm{Q}$ of a mechanical resonator is proportional to the ratio of the mechanical energy stored in the device to the dissipated energy per cycle of oscillation. The total dissipated energy is the sum of energy dissipated by a variety of physical mechanisms. Physical mechanisms of energy dissipation can be described by the two following sets of losses: extrinsic loss and intrinsic loss [19-21]. The extrinsic losses include acoustic radiation, surface loss, and air friction. The intrinsic losses include material loss, anchor loss, and thermoelastic damping. In this study, we assume that thermoelastic damping is dominant in the resonator. The physical mechanism of the thermoelastic damping can be understood as follows. When a thermally isolated elastic beam is stretched uniformly and reversibly its temperature drops. The drop in temperature compensates for the increase in entropy caused by the stress in the beam. Since the process is reversible the entropy remains constant. Similarly on compression the beam will heat up. When a structure vibrates in a more complex normal mode, there will be some regions of compression and some of extension. Depending on the timescale of the vibration heat will flow from the warmer parts 
of the structure to the cooler parts. Since heat flow is an irreversible process, this heat flow will be associated with energy loss from the vibrational mode, and corresponding damping for the resonant mode.

Fig. 1(a) shows schematic of the nanowire resonator used to investigate energy loss caused by thermoelastic damping. The cross section geometry of the beam is rectangular. The two ends of the beam are fixed and are assumed to be connected to a much larger body which acts as a thermal reservoir. Because the two ends of the beam are fixed, a boundary condition with zero displacements is set at two side surfaces of the beam. The two ends of the beam were assumed to be clamped to a body with a large thermal mass functional such as a thermal reservoir, so the isothermal boundary condition is assumed.

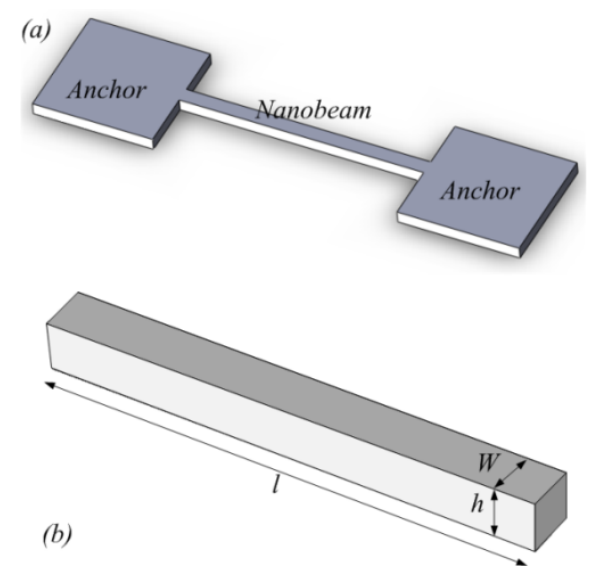

Fig. 1. Schematic of the nanowire resonator used for thermoelastic analysis with both clamped ends (a) and geometry of the modeled beam (b).

In the theory of thermoelasticity, the heat transfer equation involving thermoelastic damping is known as

$$
\rho C_{p} \frac{\partial T}{\partial t}-\nabla \cdot(\kappa \nabla T)=-\frac{E \alpha T_{0}}{1-2 v} \frac{\partial \varepsilon}{\partial t}
$$

The value and definition of parameters in Eq. (1) are shown in Table 1. The term on the right of Eq. (1) is the heat source showing the heat generation rate per unit volume. $\varepsilon$ is the elastic strain defined by

$$
\varepsilon=\varepsilon_{x}+\varepsilon_{y}+\varepsilon_{z}=\nabla \cdot u
$$

In the theory of dynamics and vibration, the equation of motion is established on the basis of the following force equilibrium:

$$
\rho \frac{\partial^{2} u}{\partial t^{2}}=\nabla \cdot \sigma
$$

where $u$ is the displacement vector, and $\sigma$ is the stress tensor.

Furthermore, according to the theory of thermoelasticity, the relation between the mechanical stress $\sigma$ and the strain $\varepsilon$ is given by

$$
\sigma=C \varepsilon-D T,
$$

where $C$ is the stiffness matrix and $\mathrm{D}$ is the thermal expansion coefficient matrix [15]. 
Table 1. Physical parameters of single crystal silicon

\begin{tabular}{|l|l|l|}
\hline Parameters & Definition & Values \\
\hline $\mathrm{E}$ & Young's modulus & $160[\mathrm{GPa}]$ \\
\hline$\alpha$ & Coefficient of thermal expansion & $2.6 \mathrm{e}^{-6}[1 / \mathrm{K}]$ \\
\hline $\mathrm{T}_{0}$ & Ambient and initial beam temperature & $300[\mathrm{~K}]$ \\
\hline$\rho$ & Density & $2330\left[\mathrm{~kg} / \mathrm{m}^{3}\right]$ \\
\hline$C_{p}$ & Specific heat capacity & $700[\mathrm{~J} /(\mathrm{kgK})]$ \\
\hline$C_{v}$ & Heat capacity $C v=\rho C_{p}$ & $1.63 \times 10^{6}\left[\mathrm{~J} /\left(\mathrm{m}^{3} \mathrm{~K}\right)\right]$ \\
\hline$\kappa$ & Thermal conductivity & $90[\mathrm{~W} /(\mathrm{m} \mathrm{K})]$ \\
\hline$v$ & Poisson's ratio & 0.28 \\
\hline
\end{tabular}

In harmonic vibration, the temperature, displacement, and velocity have the following forms

$$
\left\{\begin{array}{l}
T=\mathfrak{I}(x i) \exp (i \omega t) \\
u=U(x i) \exp (i \omega t) \\
v=V(x i) \exp (i \omega t)=i \omega U
\end{array}\right.
$$

where $\omega=i \lambda$ is the complex angular frequency, which consists of resonance and damping component; $\mathfrak{I}(x i), U(x i)$, and $V(x i)$ are the amplitudes of node temperature, node displacement, and node velocity, respectively.

By substituting Eqs. (2) and (5) into Eqs. (1) and (3) and doing mathematical procedure yields the following third-order matrix equation [14]

$$
A X=\lambda B X,
$$

where $A$ and $B$ are coefficient matrices, which are derived from applying the standard Garlerkin finite element formulation for Eq. (1), the components of matrices A and B depend on the thermal conductivity $k$, the density $\rho$, the specific heat of mass $C_{p}$, the elastic modulus $E$, the thermal expansion coefficient $\alpha$ and Poision's ratio $v$. For more detail procedure in deriving $A$ and $B$, we recommend readers to refer to Refs. [14, 15]. The eigenvector is $X=(\mathfrak{I}, U, V)^{T}$, namely a vector combining nodal temperature, displacement, and velocity together. This is a generalized eigenvalue equation. The eigenvalue of the equation is $\lambda$ which depends on the representative dimension parameters of the resonator and thermoelastic damping.

For a system with damping, the eigenvalue $\lambda$ contains information about the natural resonance angular frequency $\omega_{r}$ and the $Q$ value according to the real and imaginary parts of $\lambda$ as follows:

$$
\begin{gathered}
\omega_{r}=|\operatorname{Im}(\lambda)|, \\
Q=\frac{1}{2}\left|\frac{\operatorname{Re}(\lambda)}{\operatorname{Im}(\lambda)}\right|,
\end{gathered}
$$

which is the fraction of energy lost per radian, the factor of $1 / 2$ arising from the fact that the mechanical energy of the resonator is proportional to the square of its amplitude.

To gain information about the quality factor of the resonator, it is of interest to know its natural frequency and the $\mathrm{Q}$ value. To do this, an eigenfrequency analysis is carried out to find the eigenvalues for this system. The quality factor of the resonator is calculated by solving the 
coupled thermoelastic equations. The physical parameters of single crystal silicon beam used for simulation are shown in Table 1.

In this paper, the quality factor of the resonator is computed by finite element method in Comsol Multiphysics. COMSOL Multiphysics is a powerful interactive environment for modeling and solving all kinds of scientific and engineering problems. With COMSOL Multiphysics conventional models can easily be extended from one type of physics to multiphysics models that solve coupled physics phenomena such as thermal stress, electromechanical interaction, fluid structure interaction and so on. Especially, COMSOL Multiphysics provides a MEMS module for modeling and simulating MEMS devices such as actuators, sensors, oscillators, filters and so on. The thermoelastic damping is assumed to be the dominant damping mechanism. The coupled equations of thermoelasticity are solved within the resonator.

\section{RESULTS AND DISCUSSION}

Three lowest order flexural modes and corresponding temperature distributions in the resonator having the resonant beam with dimensions of $20 \mathrm{~nm}$ width, $20 \mathrm{~nm}$ height, and $1 \mu \mathrm{m}$ length are shown on the left and the right in Fig. 2 (a) - (c), respectively. It is due to the symmetry property of the resonant beam, so model of half beam is taken into simulation. The first order flexural mode has an eigenfrequency of $169 \mathrm{MHz}$ and a quality factor of $7.33 \times 10^{6}$. The quality factor over one million has not almost been reported for the silicon nano beam resonator operating at such high frequency in literature.

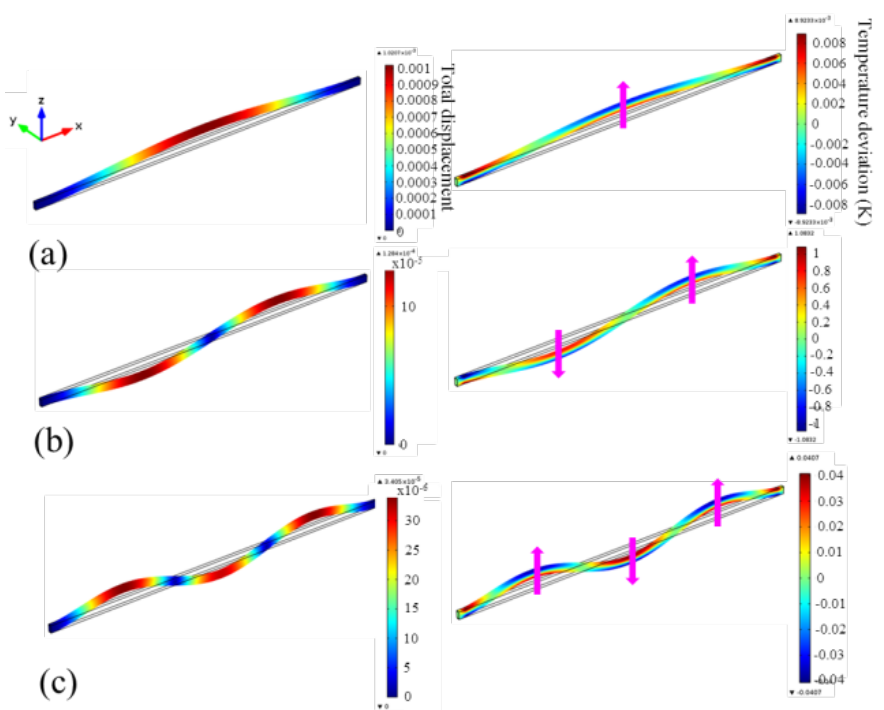

Fig. 2. The plots of eigenmode on the left from (a) - (c) and temperature distribution on the right from (a) - (c) of the clamped-clamped nanowire resonator in vertical flexural vibration. The heat flows, illustrated by the violet arrows, are caused by the temperature gradient and contribute to TED.

In comparison with the analytical result given by Zener, the quality factor of a thin isotropic beam vibrating in its fundamental mode, by considering only the thermal gradients in the direction 
of flexure, is given by [22]:

$$
Q^{-1}=\frac{E \alpha^{2} T}{\rho C_{p}} \frac{\omega_{r} \tau}{1+\left(\omega_{r} \tau\right)^{2}},
$$

where $\tau$ is the thermal relaxation time constant of the system, given by

$$
\tau=\frac{\rho C_{p} t^{2}}{\pi^{2} \kappa}
$$

The resonant angular frequency of the beam can also be computed analytically for the fundamental flexural mode and is given by:

$$
\omega_{r}=\frac{4 w}{l^{2}} \sqrt{\frac{w}{t}} \sqrt{\frac{E}{\rho}} .
$$

The physical and dimensional parameters of the silicon resonant beam in the above equations are shown in Table 1 and Fig. 1.

The fundamental resonant frequency and quality factor of the resonator calculated by Eqs. (11) and (9) are $656.69 \times 10^{6}$ and $10.62 \times 10^{6}$, respectively. Thus, the simulation results reasonably agree with the results obtained by analytical expressions given by Zener. Moreover, Young's modulus $\mathrm{E}$ in practice may be different from the value used in simulation that causes error in estimating the resonant frequency and the quality factor of the resonator. Therefore, we have examined the variation of $f$ and $Q$ while $E$ has the standard deviation of $10 \mathrm{GPa}$. From simulation results, the deviation of $f$ and $Q$ is less than $3 \%$ and $10 \%$, respectively. It is noticed that $Q$ increases when $E$ decreases. In the following we will compute characteristic parameters of the resonator by the 3D finite element method.
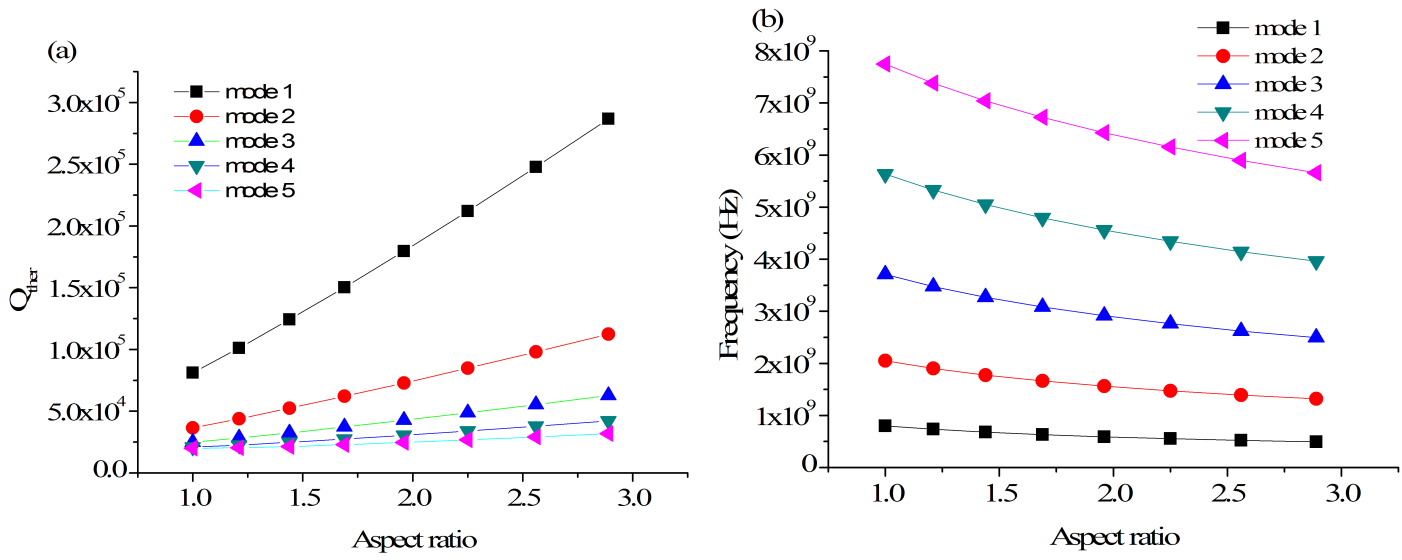

Fig. 3. (a) Quality factor and (b) resonant frequency of the nanowire resonator are calculated as a function of aspect ratio, respectively; however, the cross-section is kept to be a constant

Fig. 3 (a) - (b) shows the quality factor and the resonant frequency of modes of the nanowire resonator calculated as a function of aspect ratio (wide $w$ to thick $t$ ratio), respectively. In this case, the cross-section is $10^{4} \mathrm{~nm}^{2}$ and is kept to be constant in the investigation process. This also 
means that the beam becomes thinner when the aspect ratio increases. The quality factor increases with the aspect ratio. The fundamental mode has the $\mathrm{Q}$ value superior to different high order modes. The quality factor of the fundamental mode also increases more sharply from $81.1 \times 10^{3}$ to $286.7 \times 10^{3}$, when the aspect ratio increases from 1 to 2.89 . In contrast to the $\mathrm{Q}$ value, the frequency of the modes slightly decreases, when the aspect ratio increases. The resonant frequency is lowest to be $492 \mathrm{MHz}$ at the fundamental mode of the beam having the aspect ratio of 2.89 . The highest resonant frequency is $7.74 \mathrm{GHz}$ at the fifth flexural mode of the beam having the aspect ratio of 1 .

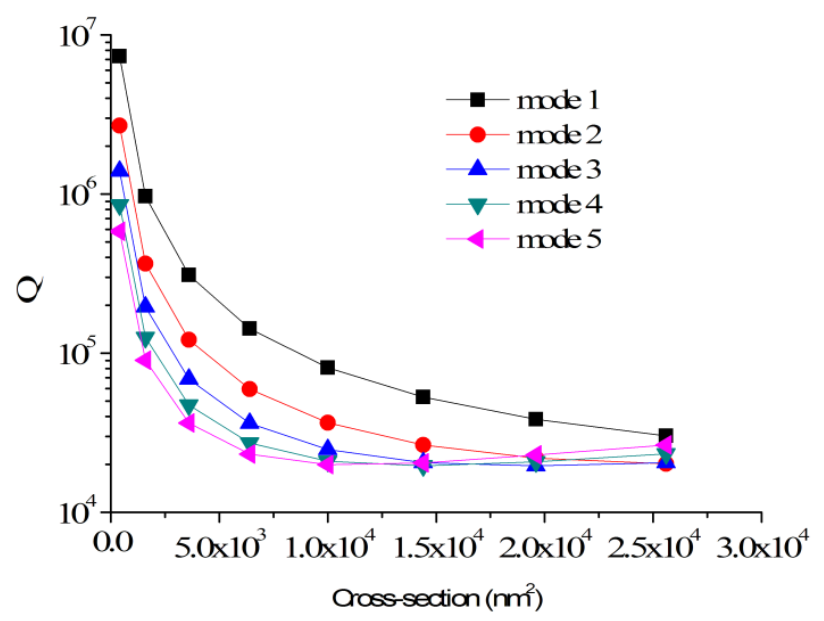

Fig. 4. Quality factor of the nanowire resonator calculated as a function of cross-section.

In order to explain for the increment of the $\mathrm{Q}$ value with the aspect ratio, we can refer to the mechanism of thermoelastic relaxation $[8,22]$. If the frequency of vibration $\omega_{r}$ is much smaller than the effective relaxation rate $1 / \tau$ of the solid, i.e. $\omega_{r} \tau \ll 1$, then the system remains essentislly in equilibrium and very little energy is dissipated. Otherwise, if $\omega_{r} \tau \gg 1$, the system has no time to relax and so energy dissipation is increased with $\omega \tau$. As an example, we consider the resonator having the fundamental mode shown in Fig. 2 (a). Using Eq. (9), the thermoelastic relaxation rate $\tau$ is evalualted to be $7.34 \times 10^{-13}$, so $\omega_{r} \tau \ll 1$. Moreover, when the aspect ratio increases, $t$ is reduced due to the cross-section to be constant in this case. As a result $\tau$ decreases with $t$. Thus, when the aspect ratio increases, the $\mathrm{Q}$ value increases due to less energy dissipated.

Fig. 4 shows the quality factor of the nanowire resonator calculated as a function of crosssection. Here, the aspect ratio of the resonant beam is 1.0. In comparison with different high order modes, the quality factor of the first order mode is also superior. As seen in Fig. 4, it is explicit that when the cross-section decreases from $2.56 \times 10^{4} \mathrm{~nm}^{2}$ to $4 \times 10^{2} \mathrm{~nm}^{2}$, the quality factor increases two orders of magnitude from $3.02 \times 10^{4}$ to $7.33 \times 10^{6}$. The resonant frequency of the first mode increases from $169 \mathrm{MHz}$ to $1.18 \mathrm{GHz}$, while that of the fifth mode increases from $2.21 \mathrm{GHz}$ to $9.33 \mathrm{GHz}$.

In the following investigation, the resonator with the cross-section of $20 \times 20 \mathrm{~nm}^{2}$ has the highest $Q$ chosen to investigate the dependence of $Q$ on the aspect ratio. In this case, the height of the beam is kept to be constant, while the width of the beam is modified. It means that the 


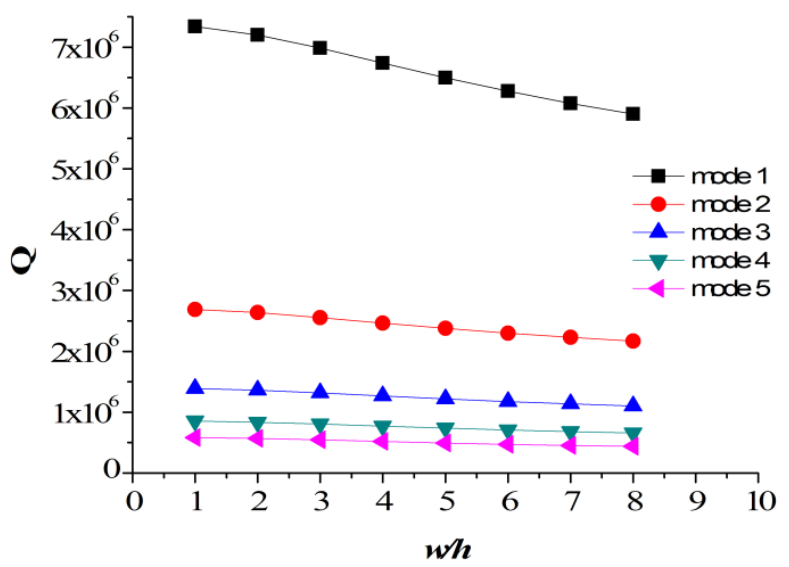

Fig. 5. Quality factor of the nanowire resonator is calculated as a function of aspect ratio. In this case, the cross-section is varied

cross-section is changed, which is contrast to that investigated above (shown in Fig. 3). Fig. 5 shows the quality factor of the nanowire resonator calculated as a function of aspect ratio. It is clear that the quality factor decreases slightly when $w / t$ increases. In this case, the frequency of the resonator is slightly reduced and less than $2 \%$ for all modes.
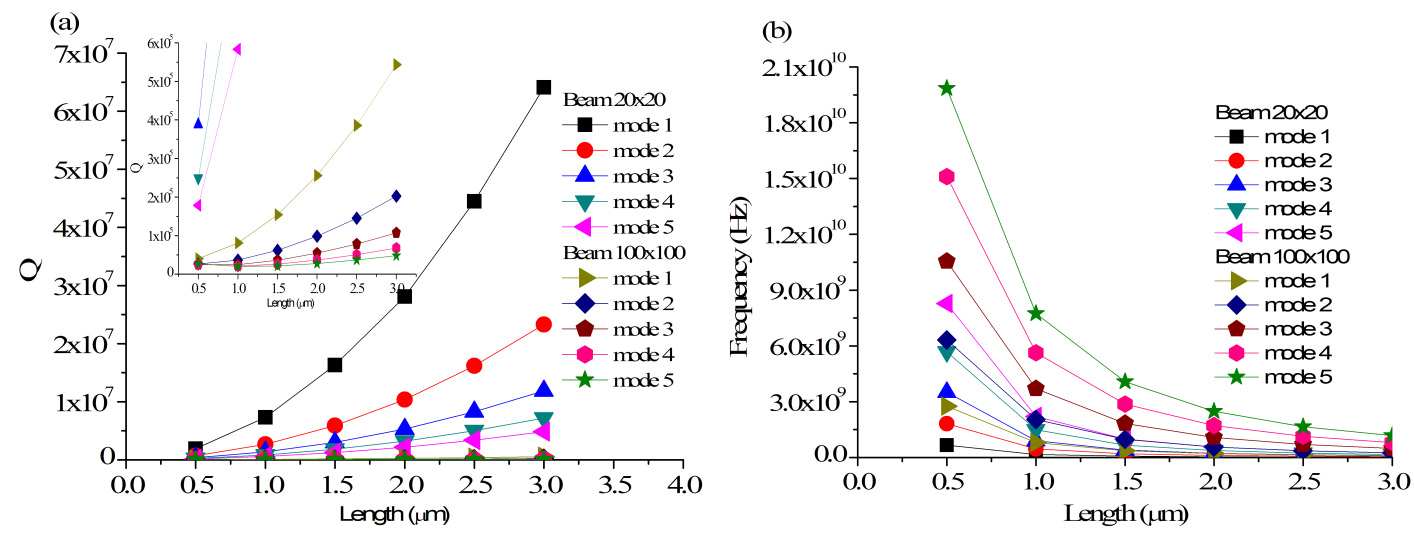

Fig. 6. (a) Quality factor and (b) resonant frequency of the nanowire resonator are calculated as functions of length for the resonant beams with cross-section of $20 \times 20 \mathrm{~nm}^{2}$ and $100 \times 100 \mathrm{~nm}^{2}$, respectively

Fig. 6 (a) - (b) shows quality factor and resonant frequency of the nano beam resonator calculated as functions of the beam length for the resonant beams with the cross-section of $20 \times$ $20 \mathrm{~nm}^{2}$ and $100 \times 100 \mathrm{~nm}^{2}$, respectively. As seen in Fig. 6 (a), it is effective to increase the Q value by increasing the length of the resonant beam. Although the resonant frequency decreases with the increment of the beam length, one resonator with the $Q$ value of $6.41 \times 10^{7}$ operating at the resonant frequency of $18.8 \mathrm{MHz}$ is feasible. With the advance of current nanotechnology, the fabrication of such resonator is possible. 


\section{CONCLUSION}

In this paper, we presented a study on the thermoelastic damping depending on operation modes of nano beam resonator. The study was carried out using finite element method. The study results show that the quality factor of the resonator is lower than at high order mode. In general, it is effective to increase the $\mathrm{Q}$ value by decreasing the cross-section area and/or increasing the length of the resonant beam. In addition, the $\mathrm{Q}$ value can be significantly improved by increasing the aspect ratio while keeping the cross-section area to be constant. The silicon nano beam resonator with the quality factor larger than one million can be achieved by decreasing the cross-section area and increasing the length of the resonant beam.

\section{ACKNOWLEDGMENT}

This research is funded by Vietnam National Foundation for Science and Technology Development (NAFOSTED) under grant number “103.02-2013.53”.

\section{REFERENCES}

[1] A.K. Naik, M.S. Hanay, W.K. Hiebert, X.L. Feng and M.L. Roukes, Nature Nanotech. 4 (2009) 445

[2] Y. Xu, J. T. Lin, B. W. Alphenaar, R. S. Keynton, Appl. Phys. Lett. 88 (2006) 143513.

[3] J. T. M. V. Beek and R. Puers, J. Micromech. Microeng., 22 (2012) 013001.

[4] M. Li, H. X. Tang, and M. Roukes, Nat. Nanotechnol. 2 (2007) 114-120.

[5] A. D. O. Connell, M. Hofheinz, M. Ansmann, R. C. Bialczak, M. Lenander, E. Lucero, M. Neeley, D. Sank, H. Wang, M. Weides, J. Wenner, John M. Martinis and A. N. Cleland, Nature, 464 (2010) 697-703.

[6] C. Doolin, P. H. Kim, B. D. Hauer, A. J. R. MacDonald and J. P. Davis, New Journal of Physics16 (2014) 035001.

[7] C. Zhang, G. Xu and Q. Jiang, J. Micromech. Microeng. 14 (2004) 1302.

[8] R. Lifshitz and M. L. Roukes, Phys. Rev. B 61 (2000) 5600-5609.

[9] G. Rezazadeh, A. Saeedi, V. Saber, T. Rezaei, C. Cetinkaya, Acta Mech 223 (2012) 1137-1152.

[10] Y. Sun, D. Fang, A.K. Soh, Int. J. Solids Struct 43 (2006) 3213-3229.

[11] Z. Hao, J. Sound Vib. 313 (1) (2008) 77-96.

[12] S. Prabhakar, S. Vengallatore, J. Micromech.Microeng. 17 (3) (2007) 532.

[13] A. Duwel, J. Gorman, M. Weinsteina, J. Borenstein, P. Ward, Sens. Actuators A: Physical, 103 (2003) 70-75.

[14] Y.B. Yi, J. Sound Vib. 309 (2008) 588-599.

[15] X. Guo, Y.-B. Yi, S. Pourkamali, Int. J. Mech. Sci. 74 (2013) 73-82.

[16] A. Duwel, R. N Candler, T. W. Kenny, M. Varghese, J. Microelectromech. Syst. 15 (6) (2006) 1437-1445.

[17] X. Guo, Y.B. Yi, J. Sound Vib. 333 (2014) 1079-1095.

[18] D. Jin, X. Li, J. Liu, G. Zuo, Y. Wang, M. Liu and H. Yu, J. Micromech.Microeng. 16 (2006) 1017.

[19] S. Schmidand C. Hierold, J. Appl. Phys. 104 (2008) 093516.

[20] S. Schmid, K. D. Jensen, K. H. Nielsen, and A. Boisen, Phys. Rev. B 84 (2011) 165307.

[21] X. Liu, S. F. Morse, J. F. Vignola, D. M. Photiadis, A. Sarkissian, M. H. Marcus, and B. H. Houston, Appl. Phys. Lett.78 (2001) 1346.

[22] C. Zener, Phys. Rev. 53 (1937) 90-9. 